\title{
Practical Simulation Application: Evaluation of Process Control Parameters in Twisted-Pair Cables Manufacturing System
}

\author{
Amr Mahfouz \\ Technological University Dublin, amr.mahfouz@tudublin.ie \\ Said Hassan \\ Cairo University, Said.Hassan@gmail.com \\ Amr Arisha \\ Technological University Dublin, amr.arisha@tudublin.ie
}

Follow this and additional works at: https://arrow.tudublin.ie/buschmarart

Part of the Business Administration, Management, and Operations Commons, and the Other Operations Research, Systems Engineering and Industrial Engineering Commons

\section{Recommended Citation}

Mahfouz, A., Hassan, S., Arisha, A.:Practical Simulation Application: Evaluation of Process Control Parameters in Twisted-Pair Cables Manufacturing System. Simulation Modelling Practice and Theory 18 (2010) 471-482.

This Article is brought to you for free and open access by the School of Marketing at ARROW@TU Dublin. It has been accepted for inclusion in Articles by an authorized administrator of ARROW@TU Dublin. For more information, please contact arrow.admin@tudublin.ie, aisling.coyne@tudublin.ie,gerard.connolly@tudublin.ie.

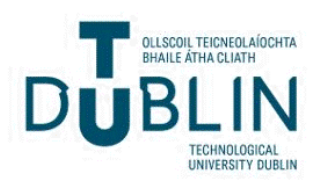




\title{
Practical Simulation Application: Evaluation of Process Control Parameters in Twisted-Pair Cables Manufacturing \\ System
}

\author{
Amr Mahfouz and Amr Arisha \\ 3S Group - College of Business \\ Dublin Institute of Technology (DIT) \\ Dublin - Ireland \\ Amr.arisha@dit.ie
}

\begin{abstract}
Intensive competition and rapid technology development in an increasingly global marketplace has left no room for competing manufacturers to harbour system inefficiencies. Moreover, customers are becoming more demanding and meeting their expectations represents an increasing challenge. TPC are used in various communication and networks hardware applications; their manufacturing facilities face many challenges through the many phases of their product life cycle including various product configurations with different equipment settings, different product flows and work in process (WIP) space limitations. The quest for internal efficiency and external effectiveness mandates that companies have to align their internal settings and resources with external requirements/orders, or in other words, significant factors must be identified prior to manufacturing process. Simulation, as one of the most flexible and powerful tools which provides a comprehensive understanding of manufacturing process variations, is increasingly aiding management and production team's decisions. An IDEF0 model in conjunction with a simulation model and a design of experiments (DOE) have been developed to characterize the Twisted-Pair Cable (TPC) production system and examine various production setting scenarios aiming to get the best product flow time.

Keywords: Twisted-Pair Cables, Simulation Modeling, Design of Experiment
\end{abstract}

\section{Introduction}

Never have the pressures on TPC manufacturers been more severe to deliver on time in the context of volatile demand from telecommunication suppliers. Moreover, global competition and technology advancement impose further complexity into the manufacturing processes of these products. TPCs have different characteristics including cable types, diameters, number of pairs and length. Each change in these characteristics influences product flow, equipment settings and product dispatching; hence creates complex interdependencies between manufacturing parameters. The manufacturing is performed in an environment of significant uncertainty both in dispatching rules used and process parameters.

Traditional analytical techniques and simple mathematical models are currently inadequate to analyze these complex manufacturing environments. Therefore, powerful modeling \& simulation technique integrated with system analysis approaches (IDEF0, DOE) are needed to properly model the dynamics as well as variability of the system and then optimize the production variables.

Simulation has proved to be a useful tool in various manufacturing applications (A.Arisha et al. 2004, Duilio Curcio et al. 2007, Darrell W.Starks et al. 2006, J.T. Lin et al. 2008 and W.Rocky Newman et al. 1999), as it is used to analyze the alternative system configurations. Many articles have been published about simulation approach in production systems, however few if any addressed TPC manufacturing issues. Therefore, 
this study is directed to analyze the influence of the changes in dispatching rules and process parameters on system performance by conducting a simulation-based full factorial design.

\section{TPC Process}

TPC process consists of four main processes; Conductor Drawing, Twisting Operation, Cable Core Jacketing and finally the Packing Process; Figure 1. The manufacturing process starts by producing the specified diameter for the conductor- the core unit of cables manufacturing. The key features of the produced conductors include, profile, diameter and surface smoothness. The drawing process is operated with four identical parallel machines to produce solid copper conductor which is surrounded by an insulating material. Pre-twisting equipments are used to prepare conductors before the twinning operation. Ahead of the twisting operation, the conductors are divided into two groups according to the specifications of the required number of pairs. Twisting operation consists of 13 machines in main five work-centers which are configured to twist any number of pairs. This process is critical as it directly affects the cable performance (transmission performance and high signal immunity). Inspection is routinely done within each process as well as a main inspection is performed after finishing twisting operation. One of three decisions is taken at the main inspection point; Accepted, Reworked or Scraped cable. Jacketing cable core process is ultimately followed to shield the conductors providing insulating and protective layer. During jacketing process, the legend and other manufacturing data are stamped on the product. At the final stage of the TPC operation, the cables are cut from the master-reel into the required length and then rolled into plastic or wooden reels as a finished product.

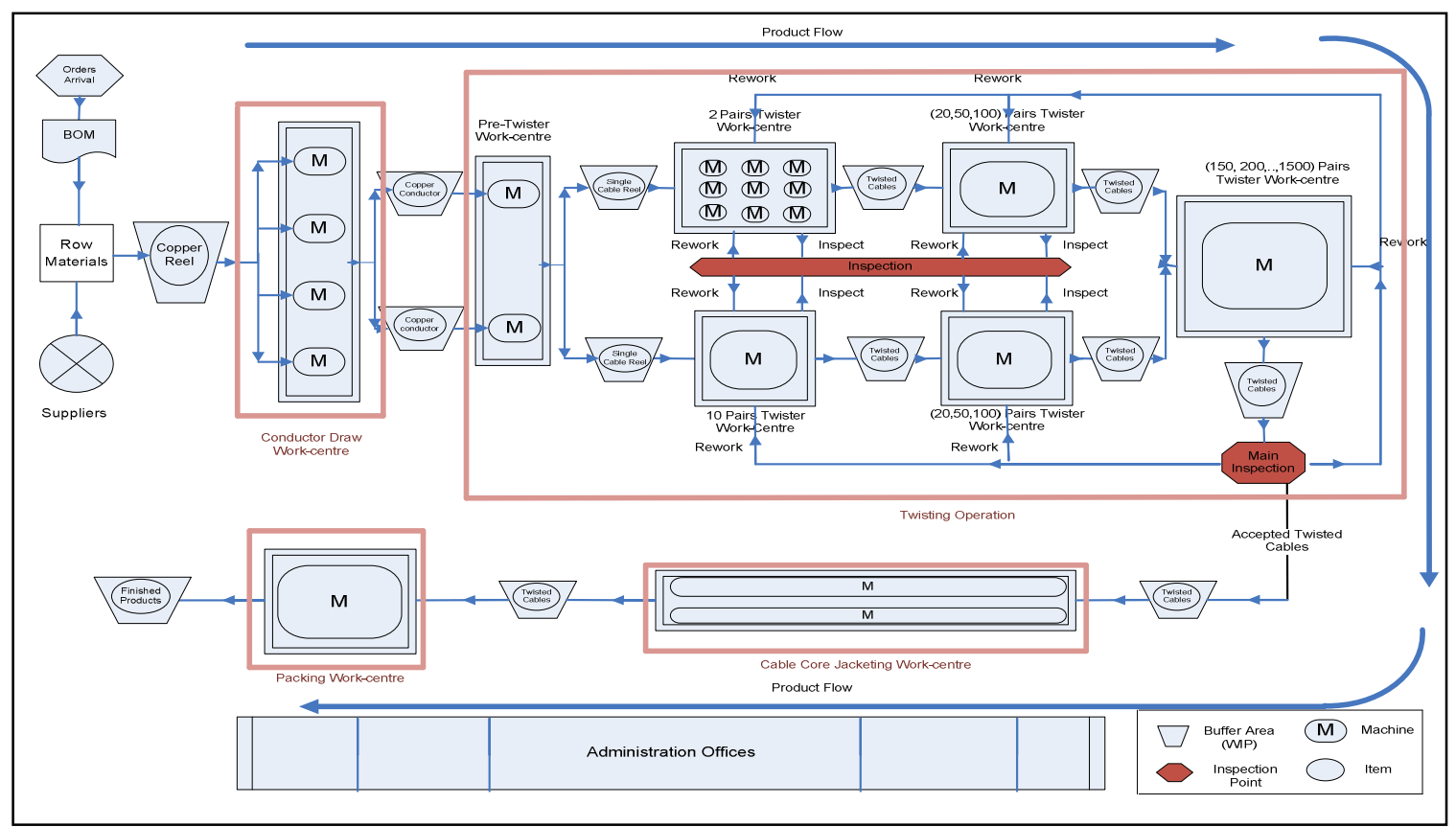

Figure 1: Schematic Diagram of TPC Factory 


\section{Problem Definition}

Given the economics of marketplace, TPC manufacturers face various challenges to meet the customized order at the right time with the right quantity. Four attributes cause wide variety for manufactured cables; (i) Cable Type, (TPC 154 and TPC 450), (ii) Cable Diameter $(0.4,0.5,0.6$ and $0.9 \mathrm{~cm}$ ), (iii) Cable Number of Pairs, (20 types varying from 2 pairs to 1500 pairs), (iv) Cable Length (dependent upon customer demand). As the number of product variants is the Cartesian product of its attribute configurations (J.C. Hernandez et al. 2007), the TPC industry has to deal with at least 160 different products for each cable length. This high-mix of products places TPC manufacturing system under significant pressure to deliver the required orders on time. Thus high WIP in limited areas, low process performance, increased setup times and high throughput time are consequential results. To complicate things more, the TPC under study does not have an efficient preplan for resources availability. Another source of complexity is the dispatching rules which need to be set prior to batch arrival.

In order to effectively manage the likes of TPC production systems, there is a need for a systematic methodology that provides a better understanding of process dynamics and to determine the optimal operating conditions. The applied model successfully integrates three analytical techniques (i.e. IDEF0, Simulation and DOE) to achieve the following objectives; (i) build an effective model to characterize TPC activities and decisions. (ii) develop a simulation model to examine TPC process performance under different production scenarios, (iii) determine the main and interaction effects of process control parameters, (vi) find the optimal combination of process parameters in order to enhance system performance. Three key process control parameters have been the focus of the study; Machine Speed, Machine Rule and Preventive Maintenance Policy. The performance measure of interest is Average Flow Time (AFT) measured by days.

\section{IDEF0 Model of TPC Process}

Taking into account the complexity of TPC manufacturing environment with such level of uncertainty due to multiple controls and mechanisms required, IDEF0 emerges as a powerful tool for modeling such intricate systems. A hierarchical modeling approach using IDEF0 allows users (e.g. strategic managers, operational engineers and system analyzers) to comprehensively understand the system and its details. An activity block which is the main unit for IDEF0 describes the main function of the process. ICOMs (Input, Control, Output and Mechanism) are represented by horizontal and vertical arrows (Figure 2). Process control (top arrow) can be company regulations, standards or legislation, whereas process mechanisms are usually the agents which facilitate the activity (e.g. People and automated tools). Further information about IDEF0 can be found in (NSIT93 and IEEE97). 
IDEF0 is used as a modeling approach to conceptualize TPC processes before developing the simulation model. Figure 3 shows the top level of the developed IDEF0 model which indicates the sequence of activities, the inputs such as sales orders and supplier list, the control such as BOMs, Due Dates and customer information, the mechanisms (several departments and computer applications) and the output (finished products). Customer order information flows through this level of IDEF0 model. The following level of the model (Figure 4) shows more details of the manufacturing activities. All production steps and their controls are well described in the diagram A3 Node (Figure 4).

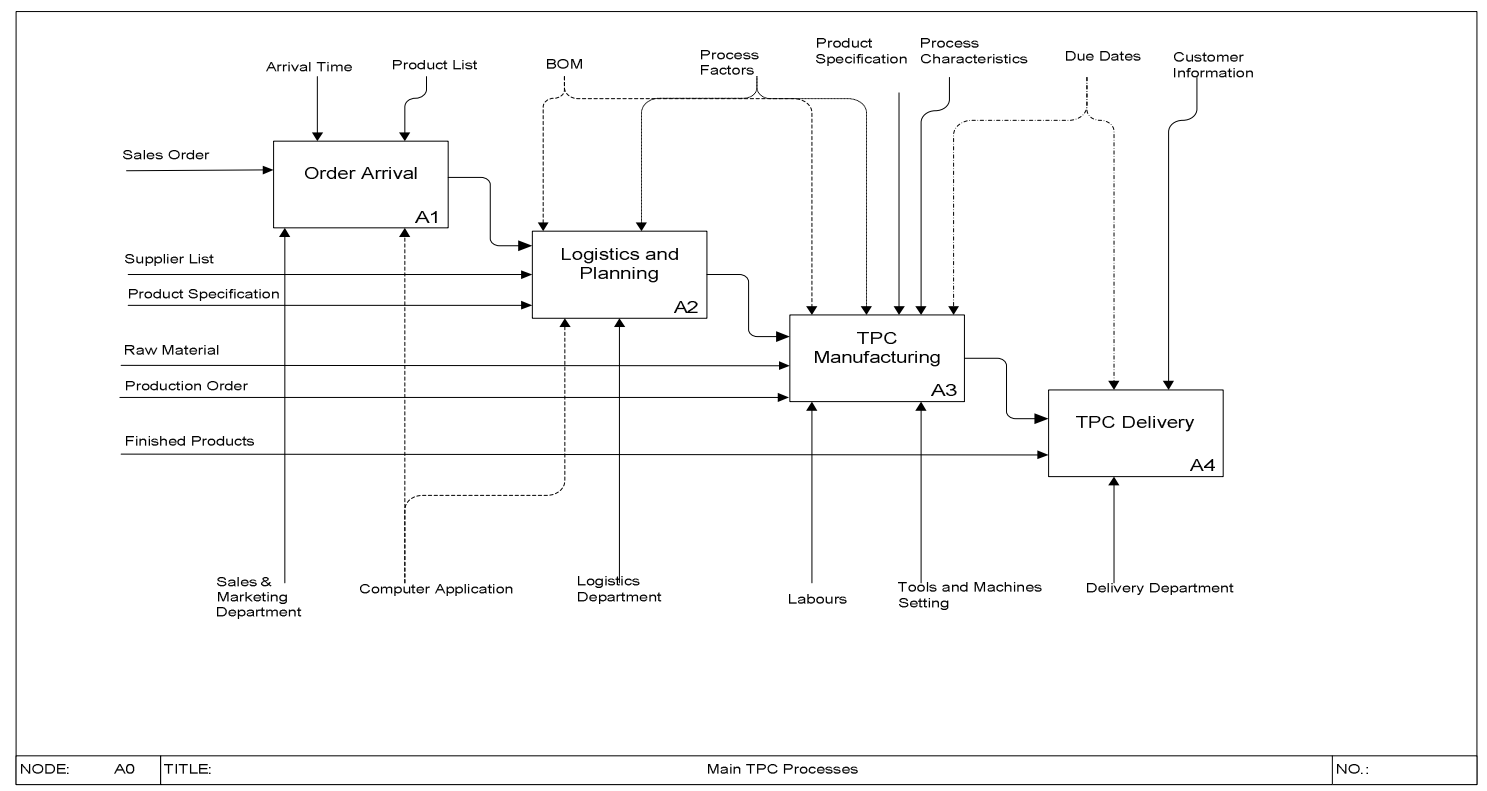

Figure 3: Basic Function Blocks for TPC

The main inputs of the manufacturing system are raw materials (i.e. copper reels) and production orders which contain required cable specifications (e.g. cable length, diameter and no. of are categorized into to the required cable Subsequently the raw group are transported workstation where operations are then admitted to a prefor a cleaning process the twisting operation.

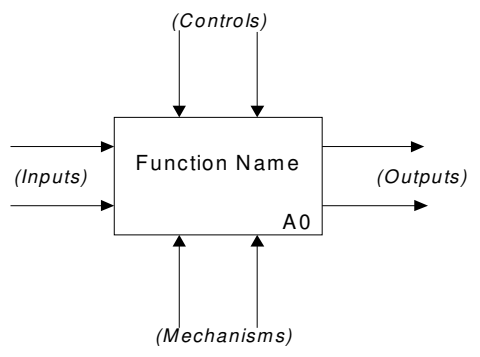

Figure 2: Basic IDEF0 Construct Pairs). These orders four groups according diameter. materials of each order to conductor drawing drawing and insulating performed. Cables are twisting workstation before they move to Operators of twisting workstation cluster the incoming cables into two groups in front of twisting 
workstations according to the required cables number of pairs. Each group follows a several route for twining operation. Cables inspection is undertaking at the main inspection unit (A35) before the commencement of jacketing and packing processes. Since the factory under study has limited production capacity and speed, cables which require the aforementioned processes may have to enter a queue, unless there is idle workstation capacity available at the moment they arrive. This means that cables which are waiting to be processed (i.e. WIP) may occupy a significant floor space until the next station is ready to process them. Subsequently, this WIP usually causes an increase in product cycle time, which has a negative effect on system performance. The variety of TPC products and their operations combined under one roof in the

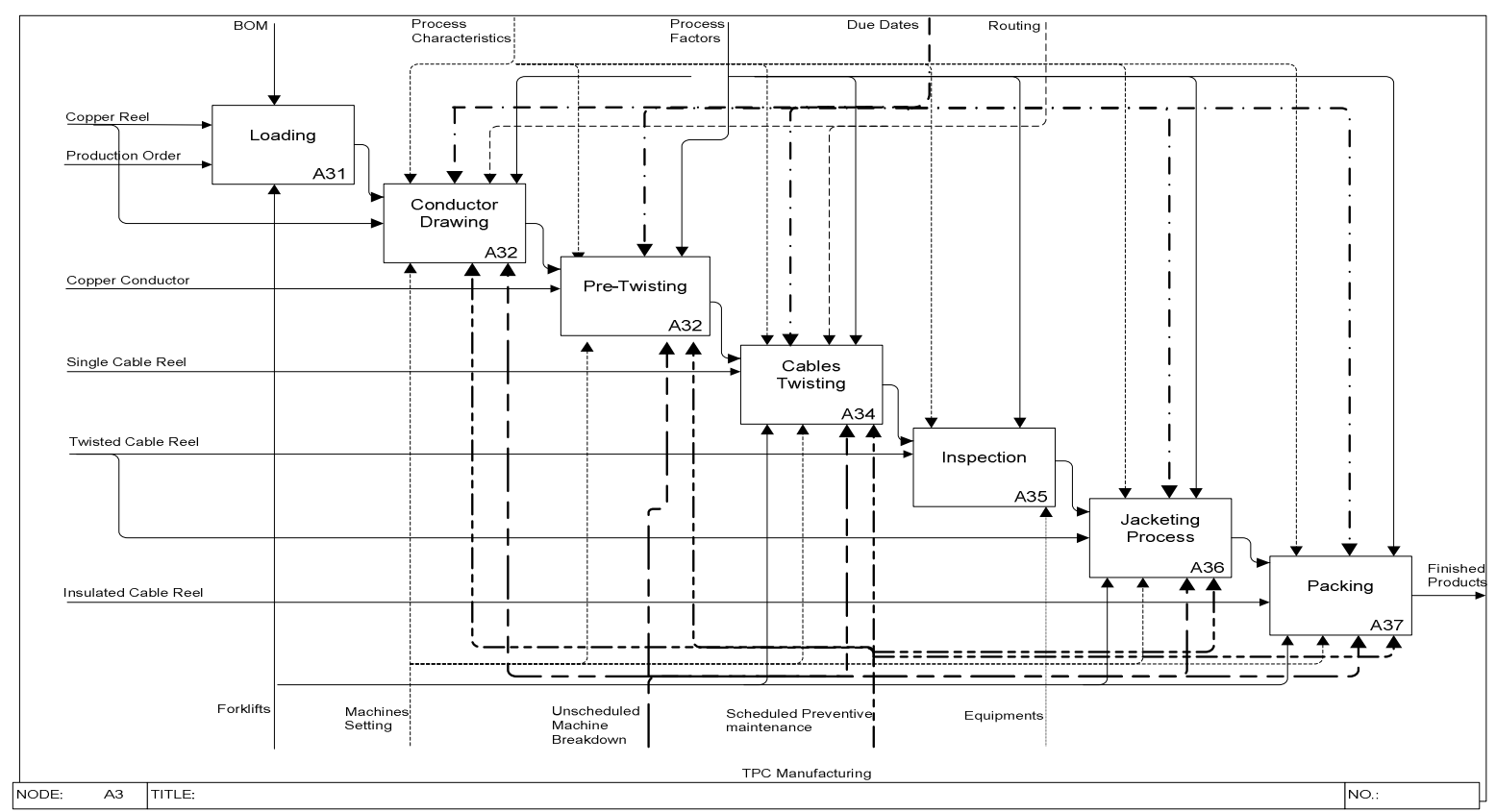

Figure 4: TPC Manufacturing Sub-Function

manufacturing system creates a very complex set of internal decisions. The establishment of process characteristics for each workstation, the routing of items and job due dates form necessary controls for TPC system.

Maintenance process represents another source of difficulty for managing production in the TPC factory. The challenge is not only how to handle maintenance problems and the resulting loss of production arising from them, but how to provide a robust preventive maintenance program in order to reduce the probability of breakdowns.

\section{Simulation Model}

In this study a probabilistic model is required in order to capture the randomness of demand patterns along with their production route, the various possible cable configurations, the variability in the length of cycle time and the uncertainty of unscheduled breakdown occurrence. The stochastic technique for discrete-event simulation is chosen as it is capable of manipulating the variability and uncertainty of this system. 
A computer simulation model based on the conceptual model shown in Figure 4 was developed. The model assumptions are (i) Product scrap and rework have been assumed as a fixed percent of production output (ii) products are interrupted on unscheduled machine breakdown occurrence (iii) preventive maintenance does not start until product processing is finished. This model uses entities to describe the cable movement through the production line, while resources represent the manufacturing tool (e.g. machines, workstations) which modifies the entities. Resources are characterized by its capacity, breakdown schedules, repair time and preventive maintenance scheme, whilst the attributes of the entities are arrival time, processing time and product configurations. Logical entities simulate the decisions for creating, joining, splitting, buffering and branching entities. Each product specification has its own statistical arrival distribution, while product processing time is a function of product diameter and length. Machine breakdown and repair time are set to have different statistical distributions for each machine. As previously mentioned, the original purpose of the model is to determine the main and interaction effect of process control parameters against three scheduling rules and to find the best combination of the process parameter to enhance system performance. Simulation model coding was done using Java \& XML technologies. That helps to provide object-oriented hierarchical and event-driven simulation capabilities for modeling large-scale applications. It also utilizes breakthrough activity-based modeling paradigm (i.e. real world activities such as assembly, batching and branching).

In an effort to make the decisions taken based on simulation models more accurate, efficient methods of verification and validation are needed. For the verification process, in addition to decomposition model (i.e. to verify every group of blocks), a simulation software built-in debugger is used. A decomposition approach is effective in the detection of errors and insuring that every block functions as expected. The studied model has been validated using two techniques. The first is 'Face Validation' that was performed by interviewing managers and manufacturing teams in order to validate simulation model results. The second approach is 'Data Comparison' which done by comparing the model output with the system output under identical input conditions.

\section{Simulation Experiments}




\begin{tabular}{|c|c|c|c|c|c|c|}
\hline Experiment & MS & MR & PM & Rule1 & Rule2 & Rule3 \\
\hline 1 & 1 & 1 & 1 & 231.64 & 156.76 & 167.47 \\
\hline 2 & 1 & 1 & 2 & 238.45 & 151.06 & 167.58 \\
\hline 3 & 1 & 2 & 1 & 171.72 & 119.57 & 106.52 \\
\hline 4 & 1 & 2 & 2 & 173.72 & 120.12 & 109.63 \\
\hline 5 & 2 & 1 & 1 & 184.92 & 134.12 & 153.52 \\
\hline 6 & 2 & 1 & 2 & 186.33 & 134.98 & 154.9 \\
\hline 7 & 2 & 2 & 1 & 142.22 & 115.68 & 103.43 \\
\hline 8 & 2 & 2 & 2 & 143.22 & 115.62 & 104.16 \\
\hline 9 & 3 & 1 & 1 & 139.02 & 117.75 & 97.5 \\
\hline 10 & 3 & 1 & 2 & 142.16 & 118 & 95.14 \\
\hline 11 & 3 & 2 & 1 & 108.15 & 99.52 & 94.73 \\
\hline 12 & 3 & 2 & 2 & 109.25 & 98.75 & 92.7 \\
\hline
\end{tabular}

TPC manufacturing system is quite complex which makes it difficult for a manager to control the process parameters which have a significant effect on system performance. In this case, a designed factorial experiment was needed to determine the relative significance of factors and their interactions in order to find the best possible combination. The studied process parameters are; Machine Speed (MS) (three different speed levels), Machine Rules (MR) (two rules) and the Preventive Maintenance Policy (PM) (two policies). The main and interaction effect of the three process parameters will be tested under various dispatching rules (Rule1, Rule2 and Rule3). For the model to reach its steady state condition, the warm-up was 4800 hours. Every simulation run represented a year of actual timing. Each experiment result (Table 1) is an average of five independent replications.

An analysis of Variance (ANOVA) model is used to study the significance of process parameters. The main and interaction effects of the chosen process parameters were analyzed using $95 \%$ confidence interval (Table $2 \& 3$ ).

It is worth noting that the main effects analysis (Table 2) is conducted by changing one single factor at a time while all other parameters are fixed. Using dispatching Rule 1, MS\& MR show significant effects on the response function with MS holding the highest $F$ value. Looking at (Table 1) results, it is clear that changes in MS impinge on AFT (44\% decreases), however changes in MR and PM decrease AFT by $24 \%$ \& $1 \%$ respectively.

Table 2: Main Effect of Process Parameters for Three Dispatching Rules

\begin{tabular}{ccccccc}
\hline Dispatching Policy & Source & Sum of Square & Df & Mean Square & $F$ & $P$ \\
\hline Rule 1 & MS & 12557.185 & 2 & 6278.593 & 8.341 & 0.009 \\
& MR & 6267.298 & 1 & 6267.298 & 4.797 & 0.043 \\
& PM & 19.918 & 1 & 19.918 & 0.01 & 0.921 \\
& & & & & & \\
Rule2 & MS & 1625.47 & 2 & 812.735 & 3.879 & 0.061 \\
& MR & 1713.869 & 1 & 1713.869 & 9.535 & 0.011 \\
& PM & 1.679 & 1 & 1.679 & 0.006 & 0.942 \\
& & & & & & \\
Rule3 & MS & 4083.728 & 2 & 2041.864 & 3.016 & 0.09 \\
& MR & 4216.388 & 1 & 4216.388 & 7.073 & 0.024 \\
& PM & 0.074 & 1 & 0.074 & 0 & 0.993 \\
\hline
\end{tabular}

Using the other two dispatching rules, MR has the greater deduction on AFT since it has the highest $\mathrm{F}$ value in both rules. Table 1 results reflect the positive effect of changing MR levels on system performance by $18 \%$ and $27 \%$ respectively. Whenever ' $\mathrm{P}$ ' value is greater than 0.05 , the parameter is not significant. PM has not shown any 
significant effect on system performance under the three selected dispatching rules. Surprisingly, MS was not influential when dispatching rule $2 \& 3$ were used.

On the other hand, interaction effect analysis is based on changing two or more factors at the same experiment to examine the impact of the changes on the response function. Table 3 shows the results of the Two-Way ANOVA model. MS\& MR interactions are significant under the three dispatching rules especially on dispatching rule 3 which has the largest $F$ value. It is clear that other interactions have low significant effect.

Table 3: Interaction Effect of Process Parameters using Three Dispatching Rules

\begin{tabular}{|c|c|c|c|c|c|c|}
\hline Dispatching Policy & Source & Sum of Square & $D f$ & Mean Square & $F$ & $P$ \\
\hline \multirow[t]{3}{*}{ Rule 1} & $M S * M R$ & 474.919 & 2 & 237.459 & 44.224 & 0.000 \\
\hline & $M S * P M$ & 5.433 & 2 & 2.716 & 0.002 & 0.998 \\
\hline & $M R * P M$ & 4.392 & 1 & 4.392 & 0.003 & 0.960 \\
\hline \multirow[t]{3}{*}{ Rule2 } & $M S$ * MR & 154.953 & 2 & 77.476 & 27.192 & 0.001 \\
\hline & $M S * P M$ & 4.882 & 2 & 2.441 & 0.008 & 0.992 \\
\hline & $\mathrm{MR}^{*} \mathrm{PM}$ & 1.548 & 1 & 1.548 & 0.007 & 0.936 \\
\hline \multirow[t]{3}{*}{ Rule3 } & $M S * M R$ & 1866.195 & 2 & 933.097 & 512.91 & 0.000 \\
\hline & $\mathrm{MS}{ }^{*} \mathrm{PM}$ & 8.454 & 2 & 4.227 & 0.004 & 0.996 \\
\hline & $M R^{*} P M$ & 0.6 & 1 & 0.6 & 0.001 & 0.978 \\
\hline
\end{tabular}

As shown in Figure 5, changes in MS level have a cogent effect on decreasing AFT. The best deduction of the response function is witnessed at level 3 of MS (Figure 5c). There is also a significant difference between the uses of the two levels of MR factor with AFT being shorter when using the second level of MR as opposed to the first one. 
Matching the aforementioned ANOVA result MR factor has shown a stronger effect on AFT then does MS factor, especially at the last two dispatching rules. For the

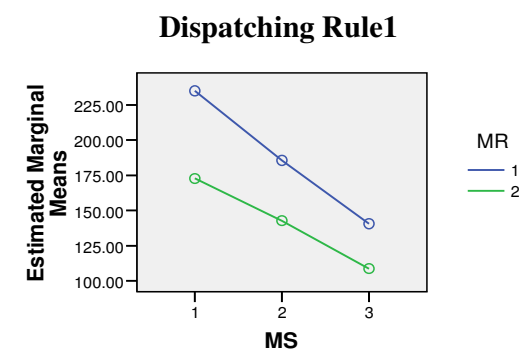

(a)

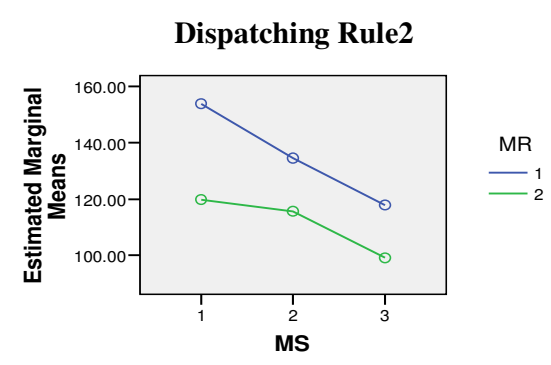

(b)

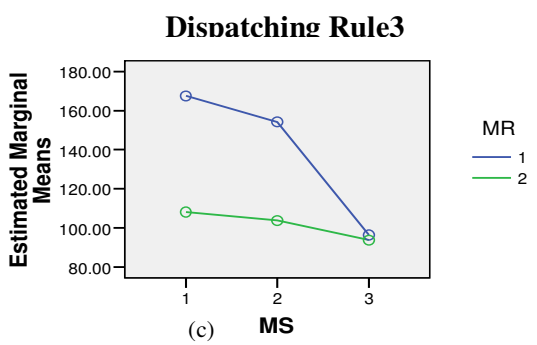

Figure 5: Average Flow Time versus the interaction between MS\&MR under Rules 1.2 and 3

three speed levels of MS, the AFT value using the second level of MR is much better than using the first level; however, the difference in performance using the two levels of MR is less pronounced the third Rule with MS at level 3 (Figure 5-c). Based on the previous analysis, the optimal factors combination at any dispatching rule is the level three of MS with the second level of MR with an AFT 108.15. Changing PM policy has a minor impact on performance function.

\section{Conclusions}

The large range of products in various configurations along with the objective of ontime delivery creates a challenging manufacturing environment for TPC. As process analysis is considered a critical task in such complex systems, it becomes necessary to choose effective approaches to model them.

IDEF0 has been used to develop the conceptual model applying standard formats to define the sequences, relationships and interdependences between TPC activities. It proves to be an efficient system description tool which offers a structured modeling approach. IDEF0 has enabled the system analysis phase by breaking the processes into stages which describe each manufacturing activity as well as envisage the overall system view.

Simulation modeling often provides a robust tool to evaluate the impact of different manufacturing policies and strategies with a minimum cost and risk. Hence, it has been employed to imitate the shop floor activities of TPC production. The significance of process parameters on system performance were analyzed using factorial design of experiments. Results show that in contrast to preventive maintenance policy, machine speed levels, machine operation rules and their interactions have significant effects on AFT regardless of dispatching rules applied.

This paper presents an integrated model of IDEF0, simulation modeling and simulation optimization (DOE) that supports decision making in TPC industry. The 
model has been verified and validated against real data and showed satisfactory results. The best combination of process control parameters (e.g. machine speed level, machine operation rules, and preventive maintenance policies) and the significance of their impact on system performance can easily be obtained as model outputs.

\section{References}

A.Arisha, P.Young, M.El Baradie.2004. 'A Simulation model to characterize the photolithography process of a semiconductor wafer fabrication'. Journal of materials Processing Technology 155-156 (2004) 2071-2079.

B.K.Rout, R.K.Mittal. 2006. 'Parametric design optimization of 2-DOF R-R planer manipulator- A design of experiment approach'. Robotics and Computer-Integrated Manufacturing 24 (2008) 239-248.

Banks, J., J. S. Carson, B. L. Nelson, and D. M. Nicol, 2005.'Discrete-event Simulation'. PrenticeHall, Upper Saddle River, NJ, New Jersey: Prentice-Hall, Inc.

Banu Y. Ekren, Arslan M. Ornek . Article in Press. 'A simulation based experimental design to analyze factors affecting production flow time'. Simulation Modelling Practice and Theory ,.

Chadrasekharan Rajendran and Oliver Holthaus. 1999. “A Comparative Study of Dispatching Rules in Dynamic Flow-shops and Job-Shops”. European Journal of Operational Research 116, 156-170.

Charles Harrel and Bruce Gladwin. 2007. " Productivity Improvement in Appliance Manufacturing”. Proceeding of the 2007 winter simulation conference.

Darrell W. Starks, Robert S. Schwieters, Daniel Creces. 2006. ‘A Decision Support Tool for Dofasco’s Primary Steelmaking Operations’. Proceedings of the 2006 Winter Simulation Conference

Duilio Curcio, Francesco Longo, Giovanni Mirabelli. 2007. 'Manufacturing process management using a flexible modeling and simulation approach'. Proceeding of the 2007 winter simulation conference.

Freddy Fuxin. 2004. 'Configurable product views based on geometry user requirements'. ComputerAided Design 37 (2005) 957-966.

Harrell, C.R, B..K. Ghosh, and R.Bowden 2000. Simulation using Promodel. McGraw-Hill.

J.C. Hernandez Matias, H. Perez Garcia, J. Perez Garcia, A.Vizan Idoipe. 2007. ‘Automatic generation of a bill of materials based on attribute patterns with variant specifications in a customer-oriented environment'. Journal of Material Processing Technology I99 (2008) 431-436.

Jose Arnaldo Barra Montevechi, Alexandre Ferreira de pinho. 2007. 'Application of Design of experiments on the simulation of a process in an automotive industry'. Proceedings of the 2007 winter simulation conference.

J.T. Lin, F.K. Wang, C.C. Peng. 2006. 'Lot release times and dispatching rule for a TFT-LCD cell process '. Robotics and Computer-Integrated Manufacturing 24 (2008) 228-238

K.K.B. Hon, S.Xu. 2007. 'Impact of product life cycle on manufacturing system reconfiguration'. Annals of the CIRP Vol. 56/1/2007.

Kelton, W. D. 1999. 'Designing Simulation Experiments'. In proceeding of the 1999 winter simulation conference 33-38. 
Law, A. M. and W. D. Kelton. 2000. 'Simulation Modeling and Analysis', $3^{\text {rd }}$ ed. McGraw-Hill, New York.

Lawrence M. Wein and Philippe B. Chevalier. 1992. "A Broader View of the Job-Shop Scheduling Problem”. Management Science, Vol.38, No.7, 1018-1033.

Nazzala, D., M., Mollaghasemi, and C. D. Anderson. 2006. 'A simulation-based evaluation of the cost of cycle time reduction in Agere system wafer fabrication facility - a case study'. International Journal of Production Economics 100: 300-313.

Osman Balci. 1997. 'Verification, Validation and Accreditation of Simulation Models'. Proceedings of the 1997 Winter Simulation Conference.

So Young Sohn, Seung Hwan Lee.2004. 'Sensitivity analysis for output performance measure in longrange dependent queuing system'. Computer \& Operations Research 31 (2004) 1527-1536

W.Rocky Newman, Mary Jo Maffei. 1999. 'Managing the job shop: Simulating the effects of flexibility, order release mechanisms and sequencing rules'. Integrated Manufacturing Systems 10/5 [1999] 266-275.

[NSIT93] "Integration Definition for Function Modeling (IDEF0)," Federal Information Processing Standards publication 183, Computer Systems Laboratory, National Institute of Standards and Technology, 1993.

[IEEE97] "Standard User Manual for the ICAM Function Modeling method IDEF0," IEEE draft standard, P1320.1.1, 1997. 